Handbook of Organic Food Processing and Production 


\title{
Handbook of Organic Food Processing and Production
}

\author{
Edited by \\ SIMON WRIGHT \\ Development Manager \\ Whole Earth Foods Ltd \\ London
}

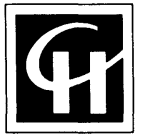

SPRINGER-SCIENCE+BUSINESS MEDIA, B.V 
First edition 1994

(C) 1994 Springer Science+Business Media Dordrecht

Originally published by Chapman \& Hall in 1994

Softcover reprint of the hardcover 1st edition 1994

Typeset in 10/12pt Times by Cambrian Typesetters, Frimley, Surrey

ISBN 978-1-4613-5877-0 ISBN 978-1-4615-2107-5 (eBook)

DOI 10.1007/978-1-4615-2107-5

Apart from any fair dealing for the purposes of research or private study, or criticism or review, as permitted under the UK Copyright Designs and Patents Act, 1988, this publication may not be reproduced, stored, or transmitted, in any form or by any means, without the prior permission in writing of the publishers, or in the case of reprographic reproduction only in accordance with the terms of the licences issued by the Copyright Licensing Agency in the UK, or in accordance with the terms of licences issued by the appropriate Reproduction Rights Organization outside the UK. Enquiries concerning reproduction outside the terms stated here should be sent to the publishers at the Glasgow address printed on this page.

The publisher makes no representation, express or implied, with regard to the accuracy of the information contained in this book and cannot accept any legal responsibility or liability for any errors or omissions that may be made.

A catalogue record for this book is available from the British Library

Library of Congress Catalog Card Number: 94-72272

@ Printed on permanent acid-free text paper, manufactured in accordance with ANSI/NISO Z39.48-1992 (Permanence of Paper). 


\section{Preface}

The increasing interest in organic foods has created a need for this book, the first practical manual to cover the processing of organic food and drink. The book shows how a company can process organic foods, emphasising economic and legal considerations. The authors have been selected for their extensive 'hands-on' experience of organic food processing. By demystifying the processing of organic foods this book will encourage those from outside the current organic food industry to become involved.

An initial introduction to organic food is followed by a review of organic legislation (chapter 2) and the organic certification process (chapter 3). The following six chapters cover the major organic commodity groups: Fruit and Vegetables, Cereal Products, Meat and Meat Products, Dairy Products, Other Processed Foods and Alcoholic Drinks. Chapter 10 is devoted to developments in the USA. Finally there is an extensive directory, giving details of the major players and organic organisations throughout the world.

The book will appeal to technical and marketing personnel in organic food and drink processing companies, as well as their counterparts in companies who want to become involved. Other people who will find this book of interest include retailers who sell organic foods; farmers who grow organic crops; lecturers and students of Food Science and Food Technology; lecturers and students of Agriculture; and anyone who wants an introduction to this rapidly developing sector of the food industry.

Simon Wright 


\section{Foreword}

For several years now, I have had much contact with organic food producers and processors. In 1981 Safeway pioneered the introduction of organic vegetables in supermarkets and four years later we achieved nationwide availability throughout our stores network.

Since their introduction in the early 1980 s, organically grown foods have seen a mixed response from the consumer. In the past few years, some of my colleagues in other retailers have decided to discontinue organic product lines, based upon the experience of the past ten years or so.

At Safeway, we remain committed to offering our customers the choice of a range of organic products. As well as the traditional fresh fruit and vegetables, Safeway offers organic wine, bread, meat and dairy products, even fromage frais.

Much of the early failure of organic produce can be traced to the uncoordinated, unstructured approach to the market, which characterised the pioneers of organics. All parts of the food supply chain must take some responsibility for this problem. Organic products must be correctly marketed so that they meet the needs of the pertinent sector of consumers. This is an important point; in the eyes of the mainstream consumer, organic products are not virtuous in their own right, so simply trying to sell them alongside traditional alternatives is not enough.

This book is important because, when one considers that a holistic approach to a process is an integral part of the organic philosophy, there has been little published which offers the coverage of this work, in an integrated fashion.

I hope that this text is useful in helping existing processors and producers in developing their organic businesses and I also hope that it may ease the passage to market for some new businesses to join the sector. An increasing number and depth of organic producers and processors is the real key to advancing the sector, because the one real limitation to potential organic customers is price, which can only be tackled by larger volumes of supply.

I am certain that Safeway will remain committed to meeting the demand of customers by offering the option of organic products for the forseeable future. 


\section{Contributors}

Edwin Broad Managing Director, Organic Connections International, Riverdale, Town Street, Upwell, Wisbech, Cambridgeshire PE14 9AF

Mark Budworth Freelance Writer, 16 Eastmayfield, Newington, Edinburgh

John Byng Head of Branch A, Agricultural Resources Policy Division, Ministry of Agriculture, Fisheries and Food, Whitehall Place (West Block), London SW1A 2HH

John Dalby Technical Director, Soil Association, 86 Colston Street, Bristol BS1 5BB

Michael Duveen Managing Director, The Farmers Dairy Company, Busses Farm, Harwood Lane, East Grinstead, West Sussex RH19 4NL

Alistair Grant Chairman, Safeway Stores plc, Hayes, Middlesex

Patrick Holden Director, Soil Association, 86 Colston Street, Bristol BS1 5BB

Ken Mergentime Writer/Editor Organic Times, New Hope Communications, 1301 Spruce Street, Boulder, Colorado 80302, USA

Michael Michaud Soil Association Inspector, Sea Spring Farm, Lyme View, West Bexington, Dorchester, Dorset DT2 9DD

Charlotte Mitchell Chairman, Soil Association, 72 Trinity Road, Edinburgh

Mark Redman Editor New Farmer and Grower, Barrow Hill Farm Cottage, Barrow Hill, Stalbridge, Sturminster Newton, Dorset DT10 2QX

Craig Sams Chairman, Whole Earth Foods Ltd, 269 Portobello Road, London W11 1LR 
Bill Starling Organic Grain Trader, William Gleadell \& Sons, Lindsey House, Hemswell Cliff, Gainsborough, Lincolnshire DN21 5TH

Simon Wright Development Manger, Whole Earth Foods Ltd, 269 Portobello Road, London W11 1LR 


\section{Contents}

1 Introduction $\quad 1$

CRAIG SAMS

1.1 Introduction

1.2 Organic farming

1.3 Conversion

1.4 Conservation grades

1.5 The cost of organic farming

1.6 How subsidies make organic farming uncompetitive

1.7 How does it work?

1.8 First World agriculture and Third World poverty

1.9 Fair trade and organic farming

1.10 History of the UK organic movement

1.11 Market size and share 13

1.12 Producers, processors and marketeers $\quad 15$

$\begin{array}{ll}\text { References } & 16\end{array}$

2 UK and European Community (EC) legislation 17 JOHN BYNG

$\begin{array}{lll}2.1 & \text { Introduction } & 17\end{array}$

2.2 Situation in the UK and the EC prior to EC legislation 17

2.3 Development of EC legislation 19

2.4 Summary of EC legislation as at 30 October $1993 \quad 20$

2.4.1 Council Regulation 2092/91 21

2.4.2 Commission Regulation 94/92 21

2.4.3 Commission Regulation 1535/92 21

2.4.4 Council Regulation 2083/92 21

2.4.5 Commission Regulation 3457/92 22

2.4.6 Commission Regulation 3713/92 22

2.4.7 Commission Regulation 207/93 22

2.4.8 Commission Regulation 1593/93 22

2.4.9 Commission Regulation 2608/93 22

2.4.10 Commission Regulation 468/94 22

2.4.11 Commission Regulation 688/94 22

2.5 Structure of the EC legislation 22

2.6 Future developments on standards 28

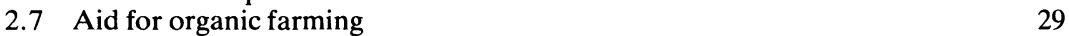

2.8 Overview 29

References $\quad 30$ 


\section{Organic certification and the importation of organically} produced foods

MICHAEL MICHAUD, MARK REDMAN and JOHN DALBY

3.1 Introduction

3.2 Organic certification and its importance 31

$\begin{array}{ll}3.3 & \text { Organic standards and certification protocol } \\ 3.43\end{array}$

3.4 Development of organic certification as a legal requirement 33

3.5 The structure of organic certification schemes in the EU

$\begin{array}{ll}3.6 & \text { Certification protocol } \\ & 38\end{array}$

3.6.1 Determination of eligibility of a business for organic certification 39

$\begin{array}{ll}\text { 3.6.2 Making an application } & 45\end{array}$

3.6.3 Inspection 46

$\begin{array}{ll}3.6 .4 \text { Post-inspection activities and certification } & 49\end{array}$

3.7 Imports of organically produced foods $\quad 51$

3.7.1 Imports from approved Third Countries 51

3.7.2 Imports from non-approved Third Countries $\quad 52$

3.8 The dynamics of organic certification $\quad 54$

Acknowledgement $\quad 55$

References $\quad 55$

4 Fruit and vegetables $\quad 56$ EDWIN BROAD

4.1 Introduction $\quad 56$

4.2 Market structure and history $\quad 57$

$\begin{array}{ll}4.3 \text { Availability } & 58\end{array}$

4.4 Growing systems compared $\quad 63$

4.4.1 Non-organic growing systems $\quad 65$

4.4.2 Organic growing systems $\quad 66$

4.5 Types of market $\quad 69$

4.5.1 Wholesale market $\quad 69$

4.5.2 Supermarkets $\quad 69$

$\begin{array}{ll}\text { 4.5.3 Manufacturing/processing } & 70\end{array}$

4.6 Imports 71

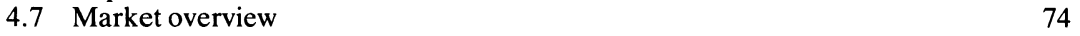

$\begin{array}{lll}4.8 & \text { The future } & 74\end{array}$

5 Cereal products $\quad 76$

BILL STARLING

$\begin{array}{lll}5.1 & \text { Principal crops } & 76\end{array}$

$\begin{array}{ll}\text { 5.1.1 Availability } & 76\end{array}$

5.1.2 Quality 76

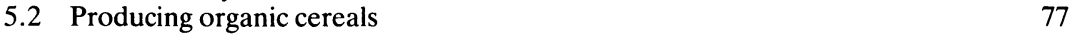

$\begin{array}{lll}5.3 & \text { Processing requirements } & 79\end{array}$

$\begin{array}{ll}\text { 5.3.1 Audit trail } & 79\end{array}$

5.3.2 Handling and processing $\quad 80$

$\begin{array}{ll}5.3 .3 \text { Ingredients } & 80\end{array}$

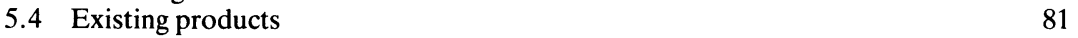

$\begin{array}{ll}5.5 \text { Summary } & 83\end{array}$ 


\section{MARK REDMAN and PATRICK HOLDEN}

6.1 Introduction

6.2 Legislation 86

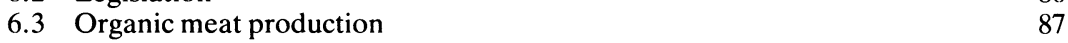

$\begin{array}{ll}\text { 6.3.1 Production standards } & 88\end{array}$

$\begin{array}{ll}\text { 6.3.2 Production systems } & 91\end{array}$

6.4 Processing and marketing organic meat $\quad 96$

6.4.1 Processing standards $\quad 96$

$\begin{array}{lr}\text { 6.4.2 Marketing } & 100\end{array}$

6.5 Some practical processing and marketing considerations 103

6.5.1 Slaughtering and cutting organic meat 104

$\begin{array}{ll}6.5 .2 \text { Retail outlets } & 105\end{array}$

$\begin{array}{ll}\text { 6.5.3 Processed foods } & 108\end{array}$

$\begin{array}{lr}\text { Acknowledgement } & 109\end{array}$

$\begin{array}{ll}\text { References } & 109\end{array}$

7 Dairy products 111

\section{MICHAEL DUVEEN}

$\begin{array}{lll}7.1 & \text { Introduction } & 111\end{array}$

$\begin{array}{ll}7.2 \text { The significance of milk } & 111\end{array}$

$\begin{array}{lll}7.3 & \text { Milk quality } & 112\end{array}$

$\begin{array}{ll}7.4 & \text { Animal husbandry on an organic dairy farm } \\ 712\end{array}$

$\begin{array}{ll}\text { 7.4.1 Organisation } & 112\end{array}$

$\begin{array}{ll}\text { 7.4.2 Living conditions } & 113\end{array}$

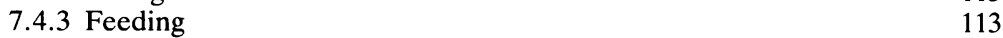

$\begin{array}{ll}\text { 7.4.4 Treating disease } & 114\end{array}$

$\begin{array}{ll}7.4 .5 \text { Breeding } & 114\end{array}$

$\begin{array}{ll}\text { 7.4.6 Weaning } & 115\end{array}$

$\begin{array}{lll}7.5 & \text { Liquid milk processing } & 115\end{array}$

$\begin{array}{ll}7.6 \text { Cheese production } & 117\end{array}$

$\begin{array}{lll}7.7 & \text { Yogurt making } & 118\end{array}$

$\begin{array}{ll}7.8 \text { Cream and butter production } & 120\end{array}$

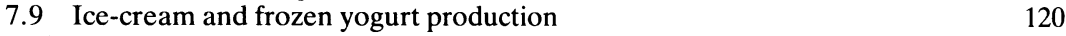

$\begin{array}{ll}7.10 \text { Marketing organic dairy products } & 121\end{array}$

$\begin{array}{ll}7.11 \text { Market development } & 122\end{array}$

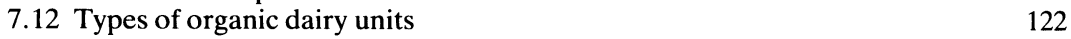

$\begin{array}{ll}\text { 7.12.1 Processing on-farm } & 123\end{array}$

$\begin{array}{ll}\text { 7.12.2 The independent dairy } & 123\end{array}$

$\begin{array}{ll}\text { 7.12.3 The specialist organic dairy } & 124\end{array}$

$\begin{array}{ll}7.13 \text { Legislation } & 124\end{array}$

$\begin{array}{ll}7.14 \text { Summary } & 125\end{array}$

8 Other organic processed foods 126 SIMON WRIGHT

$\begin{array}{ll}8.1 \text { Introduction } & 126\end{array}$

\begin{tabular}{ll}
8.2 Organic spreads & 126 \\
\hline & 126
\end{tabular}

8.2.1 Jams/pure fruit spreads $\quad 126$

$\begin{array}{ll}8.2 .2 \text { Nut butters } & 128\end{array}$

$\begin{array}{ll}\text { 8.2.3 Chocolate and carob spreads } & 130\end{array}$

$\begin{array}{ll}8.2 .4 \text { Tahini } & 130\end{array}$

8.2.5 Honey 131 
8.3 Organic juices and grain syrups 131

8.3.1 Fruit and vegetable juices 132

8.3.2 Grain-based syrups 133

8.4 Organic sauces and pickles 134

8.4.1 Sauces 134

8.4.2 Pickles and olives 135

$\begin{array}{ll}8.4 .3 \text { Mustard } & 136\end{array}$

\begin{tabular}{ll}
8.5 Organic ready meals & 136 \\
\hline & 136
\end{tabular}

8.5.1 Canned products 136

8.5.2 Frozen and chilled ready meals 138

8.5.3 Products packed in glass 138

$\begin{array}{ll}\text { 8.5.4 Baby foods } & 139\end{array}$

$\begin{array}{ll}8.6 \text { Organic snack items } & 140\end{array}$

$\begin{array}{ll}\text { 8.6.1 Savoury snacks } & 140\end{array}$

8.6.2 Chocolate 141

8.6.3 Ice-cream 143

8.7 Organic oils, vinegar and soya sauce 143

$\begin{array}{ll}\text { 8.7.1 Oils } & 143\end{array}$

$\begin{array}{ll}\text { 8.7.2 Vinegar } & 144\end{array}$

$\begin{array}{ll}\text { 8.7.3 Soya sauce } & 145\end{array}$

$\begin{array}{ll}\text { 8.8 Dried organic products } & 146\end{array}$

8.8.1 Herbs and spices $\quad 146$

$\begin{array}{ll}\text { 8.8.2 Fruit, nuts, pulses and beans } & 150\end{array}$

References $\quad 150$

9 Alcoholic drinks 151

CHARLOTTE MITCHELL and MARK BUDWORTH

9.1 Introduction 151

9.2 Consumer demand for organic wines, beers and spirits 151

9.3 Problems with non-organic alcohol 152

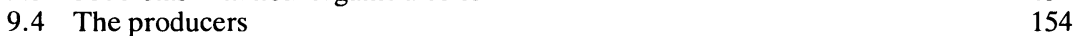

9.5 The merchants 156

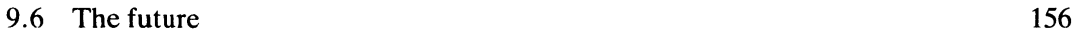

Appendix: Commonly used additives in wine-making 157

Further reading $\quad 159$

10 The USA perspective 160

KEN MERGENTIME

$\begin{array}{ll}\text { 10.1 Market overview } & 160\end{array}$

$\begin{array}{ll}\text { 10.1.1 A brief history } & 161\end{array}$

$\begin{array}{ll}10.1 .2 \text { A growing industry } & 162\end{array}$

$\begin{array}{ll}\text { 10.1.3 Some market figures } & 165\end{array}$

$\begin{array}{ll}\text { 10.1.4 Major players } & 168\end{array}$

$\begin{array}{ll}10.2 \text { Legislation } & 171\end{array}$

$\begin{array}{ll}\text { 10.2.1 Organic laws state by state } & 172\end{array}$

10.2.2 The need for national standards $\quad 175$

10.2.3 Current progress and the NOSB $\quad 176$

10.2.4 Impact of national standards on the organic industry 177

$\begin{array}{ll}10.3 \text { Future trends } & 179\end{array}$

$\begin{array}{ll}\text { 10.3.1 Product trends } & 181\end{array}$

10.3.2 Where will it sell and why? $\quad 181$

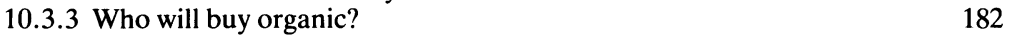

Postscript

182

References 
11 Directory of organic organisations SIMON WRIGHT

\subsection{UK}

11.1.1 Manufacturers

11.1.2 Wholesalers

11.1.3 Certifying and sector bodies

190

11.1.4 Retailers

191

11.2 International

11.2.1 Manufacturers

191

11.2.2 Distributors

191

11.2.3 Certifying and sector bodies

197

198

11.3 Trade shows

200

11.4 Other useful addresses $\quad 201$

Further reading

General index

Index of company names 